\title{
The natural history of symptomatic uncomplicated diverticular disease: a long-term follow-up study
}

\author{
Antonio Tursi ${ }^{a}$, Marilisa Franceschi ${ }^{b}$, Walter Eliseic, Marcello Picchio ${ }^{d}$, Francesco Di Marioe, \\ Giovanni Brandimarte ${ }^{f}$
}

Territorial Gastroenterology Service, ASL BAT, Andria, BT; AULSS7 Pademontana, Santorso, VI; S. Camilo Hospital, Rome; P. Colombo Hospital, ASL RM6, Velletri, Rome; University of Parma; Cristo Re Hospital, Rome, Italy

\begin{abstract}
Background Symptomatic uncomplicated diverticular disease (SUDD) affects about 20\% of patients who have diverticulosis. However, the natural history of SUDD is not yet completely understood. Our aim was to assess the outcomes of a cohort of SUDD patients during a long-term follow up.

Methods One hundred eighty-five patients suffering from SUDD were identified from a large electronic database. Symptoms assessed were abdominal pain, bloating, bowel movement/day, each of which was scored using a visual analogic scale (VAS); the symptom score was calculated by considering the value of the worst symptom present during assessment. Another VAS was used to assess patients' quality of life (QoL). Patients were treated at the physician's discretion (with rifaximin, mesalazine, probiotics, spasmolytics) only when symptoms occurred during the follow up. Follow-up visit was performed every year or whenever patients consider it necessary.

Results During the follow up (156 months, interquartile range 9-171), 47 patients were lost to follow up. Among these, 9 died from causes not related to SUDD. Acute diverticulitis occurred in 14 patients $(7.6 \%$ of the overall population): 6 patients (3.2\% of the overall population) underwent surgery, and 2 patients ( $1.1 \%$ of the overall population) died because of peritonitis. Both the symptom score and the QoL score were substantially unmodified during the study period.
\end{abstract}

Conclusions SUDD is an important disease able to affect patients significantly in the long term. Acute diverticulitis may sometimes occur in these patients, often leading to surgery with possible severe complications.

Keywords Symptomatic uncomplicated diverticular disease, acute diverticulitis, outcome, quality of life

Ann Gastroenterol 2021; 34 (1): 1-6

\begin{abstract}
aTerritorial Gastroenterology Service, ASL BAT, Andria, BT (Antonio Tursi); bDigestive Endoscopy Unit, AULSS7 "Pedemontana”, Santorso, VI (Marilisa Franceschi); 'Division of Gastroenterology, "S. Camillo" Hospital, Rome (Walter Elisei); ¿Division of Surgery, "P. Colombo" Hospital, ASL RM6, Velletri, Rome (Marcello Picchio); eDepartment of Medicine and Surgery, University of Parma, Parma (Francesco Di Mario); ${ }^{\mathrm{f} D}$ ivision of Internal Medicine and Gastroenterology, "Cristo Re" Hospital, Rome (Giovanni Brandimarte), Italy

Conflict of Interest: None
\end{abstract}

Correspondence to: Antonio Tursi, MD, Via Torino 49, 76123

Andria (BT), Italy, e-mail: antotursi@tiscali.it

Received 20 June 2020; accepted 9 September 2020;

published online 7 December 2020

DOI: https://doi.org/10.20524/aog.2020.0560

\section{Introduction}

Although colonic diverticulosis is the most frequent anatomical alteration detected during colonoscopy [1], only a minority of people who have diverticulosis may show symptoms linked to diverticula, developing the so-called "diverticular disease" (DD) [2]. Symptomatic uncomplicated DD (SUDD) of the colon is the main form of DD. It is characterized by moderate-to-severe left-lower quadrant pain, lasting more than $24 \mathrm{~h}$, not fulfilling the criteria for a diagnosis of irritable bowel syndrome (IBS), and is associated with raised levels of fecal calprotectin $[3,4]$, and fecal microbiota imbalance [5]. It is estimated that about one fifth of people who have diverticulosis develop SUDD [6], and that patients with SUDD have a wide 
range of psychological, social, and physical complaints, which impair their quality of life (QoL) [7].

However, while we know enough about the natural history of diverticulosis in terms of the risk of acute diverticulitis [8], our knowledge of the natural history of SUDD is limited. In particular, current follow-up studies, both retrospective and prospective, used a non-standardized definition of SUDD, with a follow up no longer than 5 years $[9,10]$, while the long-term risk of acute diverticulitis occurrence or need for surgery remains unknown. Moreover, there are insufficient data concerning QoL in the long-term follow up in these patients. One possible explanation is that these symptoms are often confused with IBS [11]. The aim of the present study was to assess the outcome of a cohort of SUDD patients during a long-term follow up.

\section{Patients and methods}

We conducted a retrospective study in the Azienda Unitaria Locale Socio-Sanitaria (AULSS) 7 "Pedemontana" (Veneto region), located in northeastern Italy and has 187,602 inhabitants.

\section{Selection of cases}

SUDD patients were selected using the electronic database of the Digestive Endoscopy Unit located in the Santorso Hospital (Santorso, VI - Italy), which includes patient demographics, inpatient and outpatient treatment files, and laboratory, imaging, pathology and pharmacy data. Data for analysis were obtained from a search of the electronic database of this Endoscopic Unit for the period $1^{\text {st }}$ January 2000 to $31^{\text {st }}$ December 2017, using the terms "diverticula", "diverticulosis", "diverticular disease" and "diverticulitis".

Of the patients who had diverticulosis on colonoscopy, we considered as eligible for the study only those who fulfilled the following criteria: a) they were at first diagnosis of SUDD. SUDD was defined as the presence of recurrent abdominal symptoms, in particular left lower quadrant pain lasting more than $24 \mathrm{~h}$, in patients with diverticulosis and without signs and/or symptoms and laboratory and/or endoscopic and/or radiological evidence of acute diverticulitis, in the absence of any other complication (stenosis, abscesses, fistulas), who did not fulfil the criteria for IBS $[6,12]$; b) they were not following any scheduled treatment, but were treated at the physician's discretion (with rifaximin, mesalazine, probiotics, spasmolytics); c) they were assessed every year during an annual scheduled follow-up visit. Three symptoms (left lower abdominal pain, bowel movements and bloating) were assessed at each visit using a visual analogic scale (VAS), graded from 0 (no symptom) to 10 (the most severe symptom). A symptom score was calculated by considering the value of the worst symptom present at each visit. QoL, assessed by another VAS using the question "impact of the disease on daily activities" and graded from 0 (no impact) to 10 (the most severe impact), was also reported at each visit; and d) they had given written informed consent before undergoing colonoscopy.

Patients who met any of the following criteria were excluded from the study: radiological signs (by abdominal computed tomography or ultrasound) of acute diverticulitis, complicated or uncomplicated according to well-defined criteria $[6,12]$; inflammatory bowel diseases and ischemic colitis; prior colonic resection; patients with severe liver failure (Child-Pugh class C); patients with severe kidney failure; patients with cancer, of any origin, under treatment with radio- or chemotherapy; history of alcohol, drug or chemical abuse.

\section{Outcome measurements}

After the selection of the study group, we queried the database for diverticulitis and its complications [13], using the following International Classification of Diseases, Ninth Revision, Clinical Modification (ICD-9 CM) codes:

- ICD-9-CM Code 562.10 Diverticulosis of the colon without hemorrhage;

- ICD-9-CM Code 562.11 Diverticulitis of the colon without hemorrhage;

- ICD-9-CM Code 562.12 Diverticulosis of the colon with hemorrhage;

- ICD-9-CM Code 562.13 Diverticulitis of the colon without hemorrhage;

- ICD-9-CM Code 540.0 Perforation;

- ICD-9 Code 45.74 Resection of the transverse colon;

- ICD-9 Code 45.75 Left hemicolectomy;

- ICD-9 Code 45.76 Sigmoidectomy;

- ICD-9 Code 46.04 Resection of a colonic segment;

- ICD-9 Code 48.62 Anterior resection of the rectum with concomitant colostomy;

- ICD-9 Code 48.63 Anterior resection of the rectum.

In order to make the database easier to analyze, we merged data from code 562.12 (diverticulosis of the colon with hemorrhage) and code 562.13 (diverticulitis of the colon without hemorrhage), as well as all surgical codes (ICD-9 Code 45.74, ICD-9 Code 45.75, ICD-9 Code 45.76, ICD-9 Code 46.04, ICD-9 Code 48.62 e ICD-9 Code 48.63). In this way, we obtained a complete database that included both medical and surgical complications of SUDD.

The study was approved by the Ethical Committee of the Azienda Unità Locale Socio-Sanitaria (AULSS) 7 "Pedemontana". The study protocol conformed to the ethical guidelines of the 1975 Declaration of Helsinki and was in accordance with the latest publication of 'Good Clinical Practice', as reflected in a priori approval by the institution's human research committee.

\section{Statistical analysis}

Statistical analysis was performed using MedCalc for Windows, version 18.2.1 (MedCalc Software, Mariakerke, 
Belgium). Categorical variables were expressed as absolute values and percentages, while continuous variables were expressed as median and interquartile range (IQR). We analyzed the probability of absence of diverticulitis occurrence during the follow up using the Kaplan-Meier method. The Friedman test was performed for scores. P-values $<0.05$ were considered statistically significant.

\section{Results}

According to the above criteria, a cohort of 185 SUDD patients was identified; the characteristics are reported in Table 1. The patients were followed-up for a median time of 156 (91-171) months. Forty-seven (22.7\%) patients were lost during the follow up; 9 (4.9\%) died because of causes not related to DD.

During the follow up, acute diverticulitis occurred in 14 (7.6) patients. Diverticulitis episodes occurred within 10 years from the diagnosis of SUDD; moreover, about half of them occurred between 2 and 4 years after the SUDD diagnosis (Fig. 1). Six (3.2\%) cases were affected by uncomplicated disease and were managed as outpatients. Eight (4.3\%) cases were affected by complicated disease and were managed as inpatients ( 2 patients were treated conservatively, 6 patients required surgical resection, with 2 deaths due to diffuse peritonitis).

Table 1 Characteristics of the study population

\begin{tabular}{lc}
\hline Characteristics & Study group (185 pts, \%) \\
\hline Sex, male & 67 \\
Median age, years (interquartile range) & $65.5(46-78)$ \\
\hline Age $>70$ years & $37(20)$ \\
Body mass index (BMI) & $25.9(23.3-28.9)$ \\
Obese (BMI >30) & $14(7.6)$ \\
Smoking & $43(23.2)$ \\
Symptoms & \\
Abdominal pain & $185(100)$ \\
Diarrhea & $48(25.9)$ \\
Constipation & $66(35.6)$ \\
Bloating & $21(11.3)$ \\
Main treatment on recurrence of disease & \\
Rifaximin-based & $42(24.9)$ \\
Mesalazine-based & $33(19.5)$ \\
Mesalazine + rifaximin & $18(10.6)$ \\
Rifaximin + probiotics & $14(8.3)$ \\
Mesalazine + probiotics & $11(6.5)$ \\
Probiotics alone & $22(13)$ \\
Spasmolytics & $16(9.5)$ \\
Nutraceutics & $9(5.3)$ \\
Systemic antibiotics & $4(2.4)$ \\
\hline &
\end{tabular}

With respect to the symptoms assessed during the study period, both the symptom score and the QoL score were substantially unmodified (Figs. 2 and 3). This finding also affected the need for therapeutic measures during the follow up. Only $16(8.6 \%)$ patients were asymptomatic after the diagnosis of SUDD, while $169(91.3 \%)$ patients required at least one course of medical treatment during the follow up: $16(8.6 \%)$ required only one course of treatment, $23(12.4)$ required 2 courses, $31(16.7 \%)$ required 3 courses, 27 (14.6\%) required 4 courses, 28 (13.5\%) required 5 courses, $44(23.8 \%)$ required $\geq 6$ courses of treatment.

\section{Discussion}

SUDD is a clinical entity under active debate, since little is known about its clinical and epidemiological aspects. For example, it is estimated that about $20-25 \%$ of people having diverticulosis may develop symptoms ranging from abdominal pain to change in bowel habits: what are they suffering from: IBS or SUDD? Some authors still challenge the existence of SUDD because they found no histological inflammation or changes in laboratory tests in people with diverticulosis who reported symptoms $[14,15]$. However, the occurrence of symptoms is not enough to classify a patient as suffering from SUDD [11], because SUDD has specific clinical, histological, laboratory and microbiological/metabolomic characteristics $[6,12]$. To overcome the limits of the current debate on the pathophysiology and epidemiology of SUDD, we enrolled only patients with clear evidence of SUDD $[3,4]$.

While the pathophysiology is under debate, and the real epidemiology of SUDD is still unknown, there is also a lack of knowledge about the outcomes of the disease. Only 3 studies investigated the outcomes of SUDD in terms of occurrence of acute diverticulitis and its complications, but none of them lasted longer than 5 years $[9,10,15]$. The first study, performed by Salem et al in 2007 [9], assessed 167 SUDD patients during a 5 -year follow up. Acute uncomplicated diverticulitis occurred in only 2 patients ( $1.7 \%$ of cases) and only a single patient underwent a sigmoid colectomy for recurrent symptoms. The second study was a prospective, open-label study that compared SUDD patients taking either $800 \mathrm{mg}$ of mesalamine b.i.d. for 10 days every month or no mesalazine. Gatta et al reported acute diverticulitis occurrence in 8 SUDD patients (10.4\%) not taking mesalazine during a 5-year follow up [10]. The third was a double-blind placebo-controlled study assessing the role of mesalazine, with or without probiotics, in maintaining remission in SUDD patients during a 1-year follow up [15]. The authors found that acute diverticulitis occurred in 3.5\% of patients. This was the first long-term study ever conducted in SUDD patients, and it showed that the risk of acute diverticulitis was higher and more severe than previously reported. We found that acute diverticulitis occurred in a significant percentage of patients and that half of them required surgery. Moreover, the diverticulitis risk in SUDD patients tends to decrease during long-term follow up and disappears after 10 years from the diagnosis of SUDD. These data are 


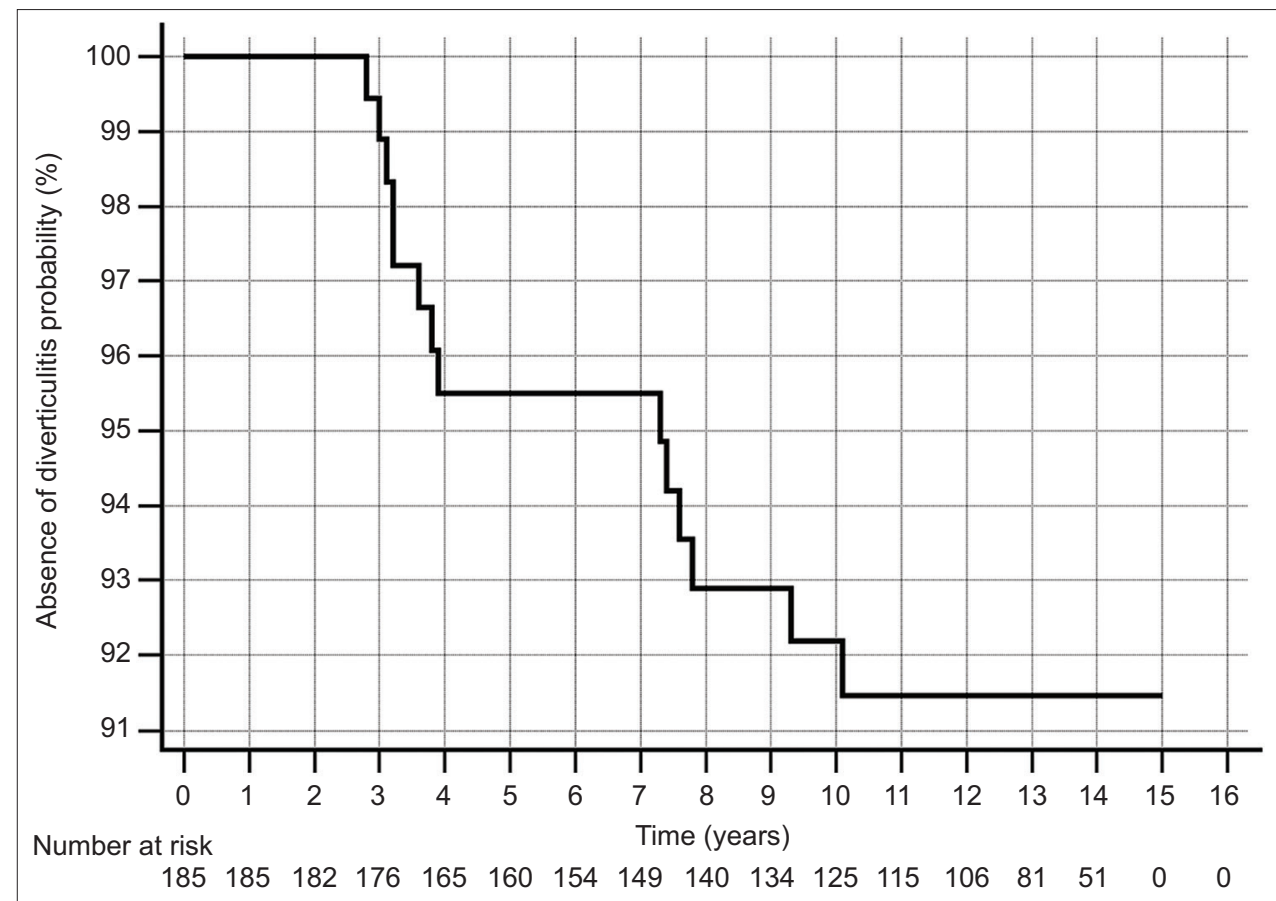

Figure 1 Kaplan-Meier analysis of cumulative rates of absence of acute diverticulitis occurrence during the follow up. Log-rank test. Diverticulitis episodes occurred within 10 years from the diagnosis of symptomatic uncomplicated diverticular disease (SUDD); moreover, about half of them occurred between 2 and 4 years after the SUDD diagnosis

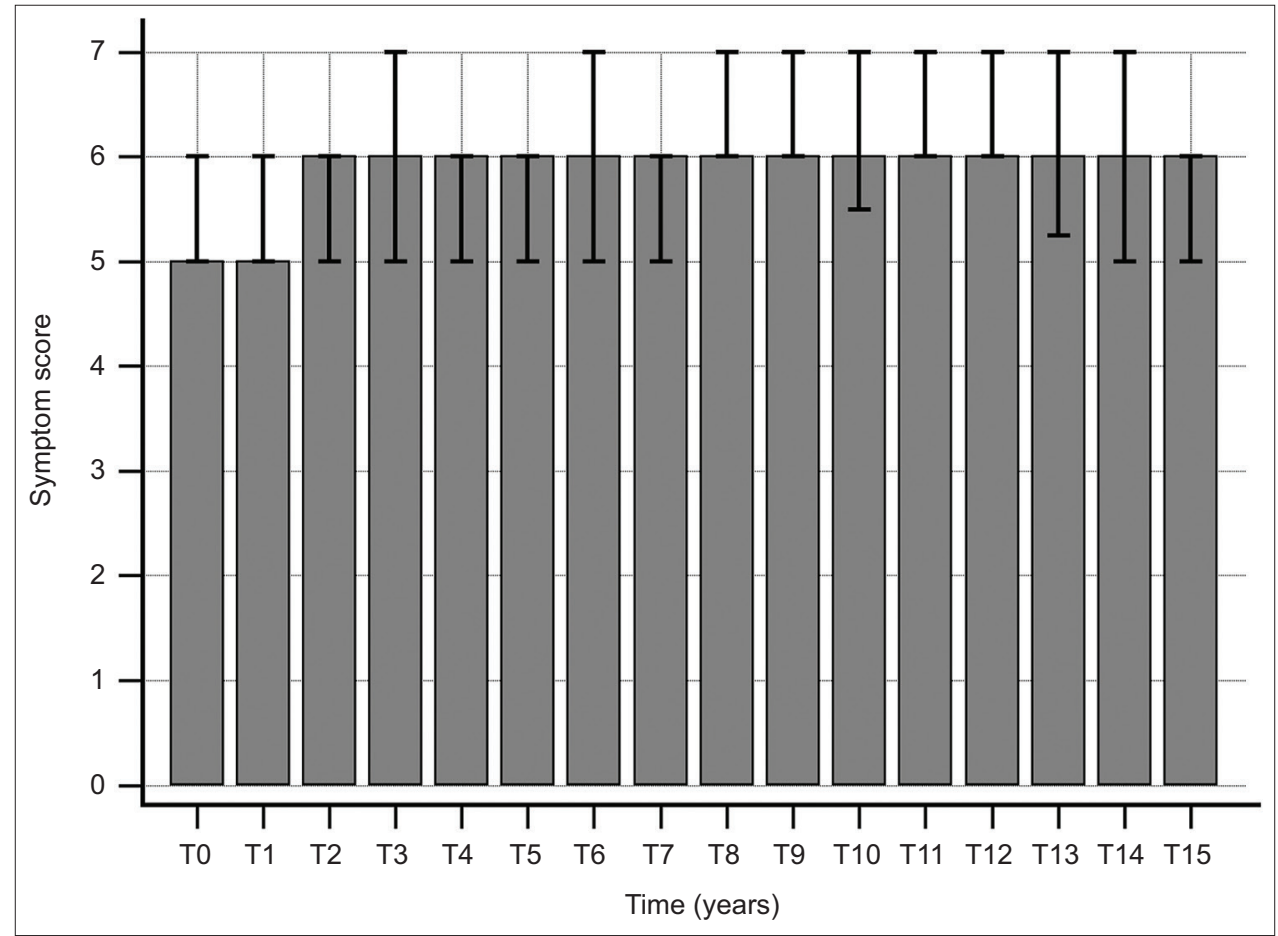

Figure 2 Symptom score at baseline and during the follow up $(\mathrm{P}=0.357)$

significantly worse than the ones reported by Salem et al [9] and by our prior experience [15], and seems to be similar to that reported by Gatta et al during a shorter follow up [10]. A possible explanation of our results may be that we selected patients using more stringent criteria. Taking all the data together, the risk of acute diverticulitis occurrence in SUDD seems to be significantly higher than the risk in people who have simple diverticulosis, in whom acute diverticulitis occurs 


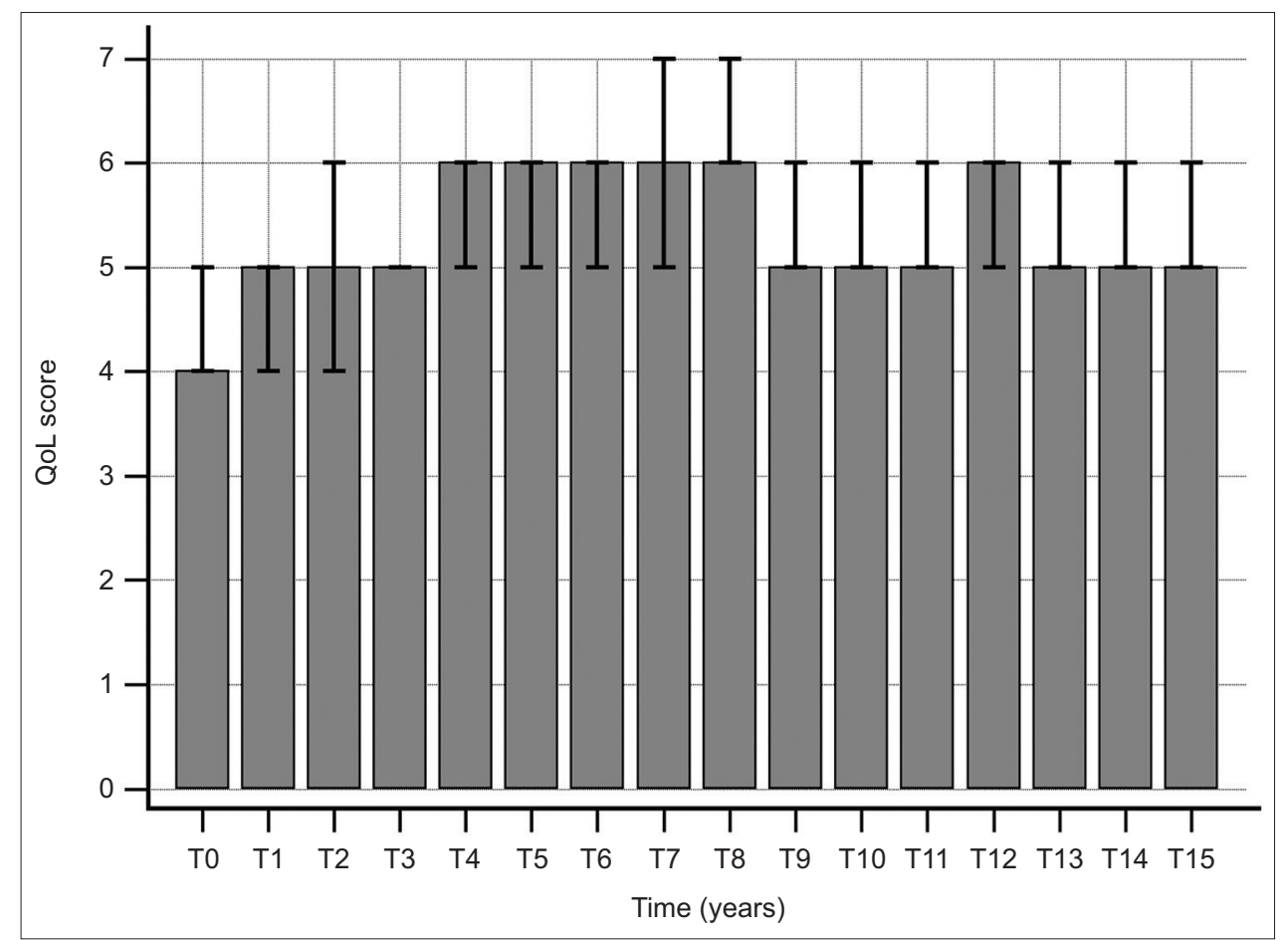

Figure 3 Quality of life (QoL) score at baseline and during the follow up $(\mathrm{P}=0.057)$

in $4.3 \%$ of cases [8]. Finally, our results suggest that SUDD also has a significant risk of complications, with a non-negligible risk of death.

Similarly, when we analyzed the recurrence/persistence of symptoms during the follow up, SUDD presented conflicting results. Salem et al reported that a large majority of their SUDD population did not have symptoms that affected their daily activities [9]. The randomized controlled trial of Tursi et al found that SUDD recurred significantly more often in the placebo group during a shorter follow up [16]. This study found that the vast majority of SUDD people had symptoms during their natural history, and that they often require more than one course of treatment. In fact, both QoL and symptoms were evenly unmodified during the entire follow up, meaning that SUDD patients had a low QoL due to persistence/ recurrence of symptoms. This is not a surprising finding, since 2 prospective studies found that QoL in SUDD patients is worse than in the general population and that it could be used as measure of the response to treatment $[7,17]$.

These results lead to 2 main considerations. The first is that SUDD is a real clinical syndrome that negatively affects patients' QoL. Although we could not use a specific questionnaire, such as the diverticulitis QoL (DV-QoL) instrument [7], because of the retrospective design of the present study, we found that symptoms were usually present during the follow up and negatively affected the QoL of our study group. This also means that SUDD patients often require recurrent therapeutic courses to control symptoms. In this way, long-term treatment is warranted, and both mesalazine and rifaximin proved to be effective in controlling long-term symptoms $[18,19]$.
The second is that those people were at high risk of developing complications (namely acute diverticulitis) with a significant mortality, and that this generally occurred during the first years after SUDD diagnosis. This behavior is similar to acute diverticulitis, in which the majority of recurrences are recorded during the first 2 years after the first episode of acute diverticulitis [20]. A possible explanation is that low-grade inflammation may play a role either in SUDD or in acute diverticulitis [21].

As expected, this study had several limitations. The first was the retrospective design, which entailed the absence of several kinds of information (for example, comorbidities or concomitant treatments). The second limit was the lacking of a standardized questionnaire to assess the QoL (such as the SF36 or DV-QoL). However, we think that the VAS scale used, specifically designed for the assessment of the impact of the disease on the daily activities, overcame this limitation adequately. The third limit was the absence of an endoscopic description of the colon in our patients, in particular according to the DICA classification. We know that this endoscopic classification may be useful for predicting the outcome of the disease and its response to therapy [22]. Once more, the cause was the retrospective design of the study, because this classification was only published in 2015 [23].

Despite the above limitations, this study showed that SUDD is an important disease, able to significantly affect patients in the long term. Acute diverticulitis may sometimes occur in those patients, often leading to surgery with possible severe complications. Further prospective studies are needed to improve our knowledge about the clinical behavior of SUDD and to find the best therapeutic approach to prevent complications in these patients. 


\section{Summary Box}

\section{What is already known:}

- Symptomatic uncomplicated diverticular disease (SUDD) affects about $20 \%$ of people who have diverticulosis

- Current follow-up studies are limited by the short follow up

- The natural history of SUDD is not yet completely understood

\section{What the new findings are:}

- A 13-year analysis was performed on a SUDD population

- During the follow up, the majority of patients had persistent symptoms associated with a low quality of life; about $8 \%$ of those patients developed acute diverticulitis, and $1.1 \%$ died from diverticulitis complications

- This study confirms that SUDD is a clinical syndrome that significantly affects the patient during the long term

\section{References}

1. Everhart JE, Ruhl CE. Burden of digestive diseases in the United States part II: lower gastrointestinal diseases. Gastroenterology 2009; 136:741-754.

2. Tursi A. Diverticulosis today: unfashionable and still underresearched. Therap Adv Gastroenterol 2016;9:213-228.

3. Cuomo R, Barbara G, Andreozzi P, et al. Symptom patterns can distinguish diverticular disease from irritable bowel syndrome. Eur J Clin Invest 2013;43:1147-1155.

4. Tursi A, Elisei W, Picchio M, Giorgetti GM, Brandimarte G. Moderate to severe and prolonged left lower-abdominal pain is the best symptom characterizing symptomatic uncomplicated diverticular disease of the colon: a comparison with fecal calprotectin in clinical setting. J Clin Gastroenterol 2015;49:218-221.

5. Tursi A, Mastromarino P, Capobianco D, et al. Assessment of fecal microbiota and fecal metabolome in symptomatic uncomplicated diverticular disease of the colon. J Clin Gastroenterol 2016;50 Suppl 1:S9-S12.

6. Tursi A, Scarpignato C, Strate LL, et al. Colonic diverticular disease. Nat Rev Dis Primers 2020;6:20.
7. Spiegel BM, Reid MW, Bolus R, et al. Development and validation of a disease-targeted quality of life instrument for chronic diverticular disease: the DV-QOL. Qual Life Res 2015;24:163-179.

8. Shahedi K, Fuller G, Bolus R, et al. Long-term risk of acute diverticulitis among patients with incidental diverticulosis found during colonoscopy. Clin Gastroenterol Hepatol 2013;11:1609-1613.

9. Salem TA, Molloy RG, O’Dwyer PJ. Prospective, five-year followup study of patients with symptomatic uncomplicated diverticular disease. Dis Colon Rectum 2007;50:1460-1464.

10. Gatta L, Di Mario F, Curlo M, et al. Long-term treatment with mesalazine in patients with symptomatic uncomplicated diverticular disease. Intern Emerg Med 2012;7:133-137.

11. Tursi A, Scarpignato C. Symptomatic uncomplicated diverticular disease: chronic abdominal pain in diverticulosis is not enough to make the diagnosis. Clin Gastroenterol Hepatol 2018;16:2001-2002.

12. Tursi A, Brandimarte G, Di Mario F, et al. International Consensus on Diverticulosis and Diverticular Disease. Statements from the 3rd International Symposium on Diverticular Disease. J Gastrointestin Liver Dis 2019;28:57-66.

13. https://www.cdc.gov/nchs/icd/icd9cm.htm

14. Peery AF, Keku TO, Addamo C, et al. Colonic diverticula are not associated with mucosal inflammation or chronic gastrointestinal symptoms. Clin Gastroenterol Hepatol 2018;16:884-891.

15. Järbrink-Sehgal ME, Rassam L, Jasim A, et al. Diverticulosis, symptoms and colonic inflammation: a population-based colonoscopy study. Am J Gastroenterol 2019;114:500-510.

16. Tursi A, Brandimarte G, Elisei W, et al. Randomised clinical trial: mesalazine and/or probiotics in maintaining remission of symptomatic uncomplicated diverticular disease-a double-blind, randomised, placebo-controlled study. Aliment Pharmacol Ther 2013;38:741-751.

17. Comparato G, Fanigliulo L, Aragona G, et al. Quality of life in uncomplicated symptomatic diverticular disease: is it another good reason for treatment? Dig Dis 2007;25:252-259.

18. Tursi A, Di Mario F, Brandimarte G, et al. Intermittent versus everyday mesalazine therapy in preventing complications of diverticular disease: a long-term follow-up study. Eur Rev Med Pharmacol Sci 2013; 17:3244-3248.

19. Di Mario F, Miraglia C, Cambiè G, et al. Long-term efficacy of rifaximin to manage the symptomatic uncomplicated diverticular disease of the colon. J Investig Med 2019;67:767-770.

20. Eglinton T, Nguyen T, Raniga S, Dixon L, Dobbs B, Frizelle FA. Patterns of recurrence in patients with acute diverticulitis. Br J Surg 2010;97:952-957.

21. Tursi A, Elisei W. Role of inflammation in the pathogenesis of diverticular disease. Mediators Inflamm 2019;2019:8328490.

22. Tursi A. Endoscopic diagnosis of diverticulosis and diagnosis of symptomatic uncomplicated diverticular disease of the colon: if you properly classify, you properly make the diagnosis. Am J Gastroenterol 2019;114:1349-1350.

23. Tursi A, Brandimarte G, Di Mario F, et al. Development and validation of an endoscopic classification of diverticular disease of the colon: the DICA classification. Dig Dis 2015;33:68-76. 